\section{ORIGINAL RESEARCH}

\author{
O. Lindberg \\ P. Östberg
}

B.B. Zandbelt

J. Öberg

Y. Zhang

C. Andersen

J.C.L. Looi

N. Bogdanović

L.-O. Wahlund

\title{
Cortical Morphometric Subclassification of Frontotemporal Lobar Degeneration
}

BACKGROUND AND PURPOSE: Frontotemporal lobar degeneration (FTLD) is a primary neurodegenerative disease comprising 3 clinical subtypes: frontotemporal dementia (FTD), semantic dementia (SD), and progressive nonfluent aphasia (PNFA). The subdivision is primarily based on the characteristic clinical symptoms displayed by each subtype. We hypothesized that these symptoms would be correlated to characteristic patterns of brain atrophy, which could be indentified and used for subclassification of subjects with FTLD.

MATERIALS AND METHODS: Volumes of 9 cortical regions were manually parcellated and measured on both hemispheres on 27 controls, 12 patients with FTD, 9 patients with PNFA, and 13 patients with $\mathrm{SD}$. The volumetric data were analyzed by traditional $t$ tests and by a multivariate discriminant analysis (partial least squares discriminant analysis).

RESULTS: The ensemble or pattern of atrophy was a good discriminator in pair-wise comparison between the subtypes: FTD compared with SD (sensitivity 100\% [12/12], specificity 100\% [13/13]); FTD compared with PNFA (sensitivity 92\% [11/12], specificity 89\% [8/9]); and SD compared with PNFA (sensitivity $86 \%$ [11/13], specificity 100\% [9/9]). Temporal-versus-frontal atrophy was the most important pattern for discriminating SD from the other 2 subtypes. Right-sided versus left-sided atrophy was the most important pattern for discriminating between subjects with FTD and PNFA.

CoNCLUSIONS: FTLD subtypes generally display a characteristic pattern of atrophy, which may be considered in diagnosing patients with FTLD.
$\mathbf{F}$ rontotemporal lobar degeneration (FTLD) remains a riddle 80 years after its conception. ${ }^{1}$ The international consensus criteria for FTLD describe 3 clinical subtypes: frontotemporal dementia (FTD), progressive nonfluent aphasia (PNFA), and semantic dementia (SD), believed to result from characteristic patterns of cortical atrophy. ${ }^{2}$ FTD is characterized by early changes in personality and comportment, whereas aphasia is absent at onset. PNFA refers to progressive deficits in speech production, including anomia and apraxia of speech, phonologic paraphasias, or agrammatism, though word comprehension and comportment are initially preserved. SD is defined by fluent speech, gradually progressive anomia, and impaired word comprehension combined with associative agnosia or prosopagnosia. In SD, surface alexia and lexical agraphia can be demonstrated in patients who use alphabetic writing systems. Behavioral changes, such as obsessions and excessive thrift, also occur in SD.

Clinical syndromes overlap to some extent. For example,

Received December 3, 2008; accepted after revision January 16, 2009.

From the Department of Neurobiology, Care Science and Society (O.L., P.Ö., B.B.Z., C.A., N.B., L.-O.W.), Section of Clinical Geriatrics, Karolinska Institute, Karolinska University Hospital Huddinge, Stockholm, Sweden; Department of Clinical Science, Intervention and Technology (J.Ö., Y.Z.), Karolinska Institute, Stockholm, Sweden; Academic Unit of Psychological Medicine (J.C.L.L.), Australian National University Medical School, Canberra, Australia. Dr. Zandbelt is now with the Department of Psychiatry, Rudolf Magnus Institute of Neuroscience, University Medical Center Utrecht, the Netherlands.

This work was supported by the Swedish Alzheimer Foundation, Swedish Brain Power, and the Swedish research council. P.Ö. was supported by grants from Aina Börjeson's Foundation for Speech-Language Pathology Research and Treatment and from the Gun and Bertil Stohne Foundation.

Please address correspondence to Lars-Olof Wahlund, MD, Division of Clinical Geriatrics, Department of Neurobiology, Health Care Science and Society, Karolinska Institute, Novum Science Park, Floor 5, SE-14186 Stockholm, Sweden; e-mail: lars-olof.wahlund@ki.se

Indicates open access to non-subscribers at www.ajnr.org

DOI 10.3174/ajnr.A1545 patients with FTD may develop features of PNFA, and those with SD may share behavioral features with FTD. This overlap is 1 argument for considering FTLD or Pick complex as a unitary consistent clinical entity. ${ }^{3}$

Although variability may be found within each FTLD subtype, the subtypes are generally considered to arise from distinct cortical basic regional patterns of atrophy (BRPA), which, in turn, cause dysfunction in cognition, emotions, and behavior associated with such regions. ${ }^{2}$ We have focused on cortical BRPA. Few studies have directly compared the BRPA in all FTLD subtypes. ${ }^{4-6}$ Using voxel-based morphometry, Grossman et $\mathrm{al}^{4}$ found characteristic BRPA differences between all FTLD subtypes. Some differences were, however, expressed as rather small subsections within an anatomically defined area.

Kipps et $\mathrm{al}^{5}$ used an MR imaging-based visual rating scale to compare a large sample of patients with FTLD (51 with FTD, 22 with PNFA, and 52 with SD). The assumption that each FTLD subtype displays a BRPA was seriously challenged by their results, because only $53 \%$ of patients with FTD and $71 \%$ of patients with PNFA showed any clear pathology on the scans. Moreover, a few patients with SD had more frontal than temporal atrophy, and 2 patients with PNFA had right-hemisphere-predominant atrophy. It has been suggested that some individuals who meet the current criteria for FTD may not have a neurodegenerative disease at all. ${ }^{7}$

Due to the inconsistency in previous findings, the purpose of this study was to further investigate the correlation between symptoms and brain atrophy in FTLD subtypes and to test to what extent patterns of atrophy could be defined and used for subclassification of subjects with FTLD into subtypes. To study this, we investigated MR imaging scans of FTLD cases collected during approximately 10 years at the Memory Clinic, Karolinska University Hospital Huddinge, Stockholm, Sweden. 


\begin{tabular}{|c|c|c|c|c|}
\hline & Control & FTD & SD & PNFA \\
\hline$\overline{\text { No. }}$ & 27 & 12 & 13 & 9 \\
\hline $\operatorname{Sex}(M / F)$ & $7: 20$ & $3: 10$ & $5: 9$ & $3: 6$ \\
\hline Age (yr) & $61.1(53-78)$ & $59.5(42-72)$ & $63.8(52-77)$ & $64.9(57-78)$ \\
\hline MMSE & 28.7 (25-30) & $20.8(10-30)^{*} \dagger$ & $22.9(5-29)^{*} \dagger$ & $22.5(15-28)^{*} \ddagger$ \\
\hline $\begin{array}{l}\text { Disease } \\
\quad \text { duration (yr) }\end{array}$ & - & $1.7(0.3-3.4)$ & $3.9(1.3-7.7)^{*} \S$ & $3.6(0.1-8.1)$ \\
\hline
\end{tabular}

Note:-MMSE indicates Mini-Mental State Examination; -, no data; Disease duration, duration between the appearance of the first symptom of the disease to scanning time; FTD, frontotemporal dementia; SD, semantic dementia; PNFA, progressive nonfluent aphasia.

${ }^{*}$ Kruskal-Wallis test.

$\dagger P<.01$ compared with controls.

$\ddagger P<.05$ compared with controls.

$\S P<.01$ compared with FTD.

\section{Materials and Methods}

\section{Subjects}

Participants were recruited retrospectively from the Memory Clinic at the Karolinska University Hospital. All subjects went through the standard investigation procedure for patients referred to the memory clinic, which includes a medical examination and a standard psychometric test battery. The clinical diagnosis was determined at a multidisciplinary consensus conference with physicians, neuropsychologists, speech-language pathologists, and nurses. Table 1 summarizes the demographic data of the included patients. Only patients with a primary degenerative cerebral process were selected, excluding patients with signs of cerebrovascular or systemic disorders. Patients with FTLD at different stages of the disease were included. Diagnoses for dementia were based on criteria of the ICD-10 (International Classification of Diseases, Tenth Revision), whereas the subtypes of FTLD were based on international consensus criteria. ${ }^{2}$

The control group comprised individuals who had been referred to the Memory Clinic because of mild subjective forgetfulness in everyday life. Objective impairment was ruled out through comprehensive neuropsychological assessment (impairment was defined as performance $\geq 1.5$ SD unit below the mean on any cognitive test). To further minimize the risk of including participants with neurodegenerative diseases in very early stages, we included only those participants whose performance did not deteriorate over a minimum of 2-years follow-up. Patients with dementia were also followed through the progression of the disease.

There were a total of 61 subjects: 34 patients with FTLD (12 with FTD, 13 with SD, 9 with PNFA), and 27 controls.

\section{Image Acquisition}

T1-weighted MR images were acquired on a 1.5T Magnetom Vision Plus scanner (Siemens Medical Systems, Erlangen, Germany). A 3D magnetization-prepared rapid gradient echo pulse sequence (TR, $11.4 \mathrm{~ms}$; TE, $4.4 \mathrm{~ms}$; TI, $300 \mathrm{~ms}$; flip angle, $10^{\circ}$; NEX, 1) was used to obtain 72 contiguous coronal $2.5-\mathrm{mm}$ sections with a $512 \times 144$ matrix and a 230-mm FOV.

\section{Cortical Parcellation and Volumetry}

The software program MRIcro (Version 1.37; Rorden and Brett, 2000, available at: http://www.sph.sc.edu/comd/rorden/mricro.html) was used for parcellation of the cortex. With this software, an image can be viewed in horizontal, sagittal, and coronal directions simultaneously with a reconstruction of the surface of the brain.

Measurements were subsequently performed on the HERMES MultiModality software package (Nuclear Diagnostics, Stockholm,
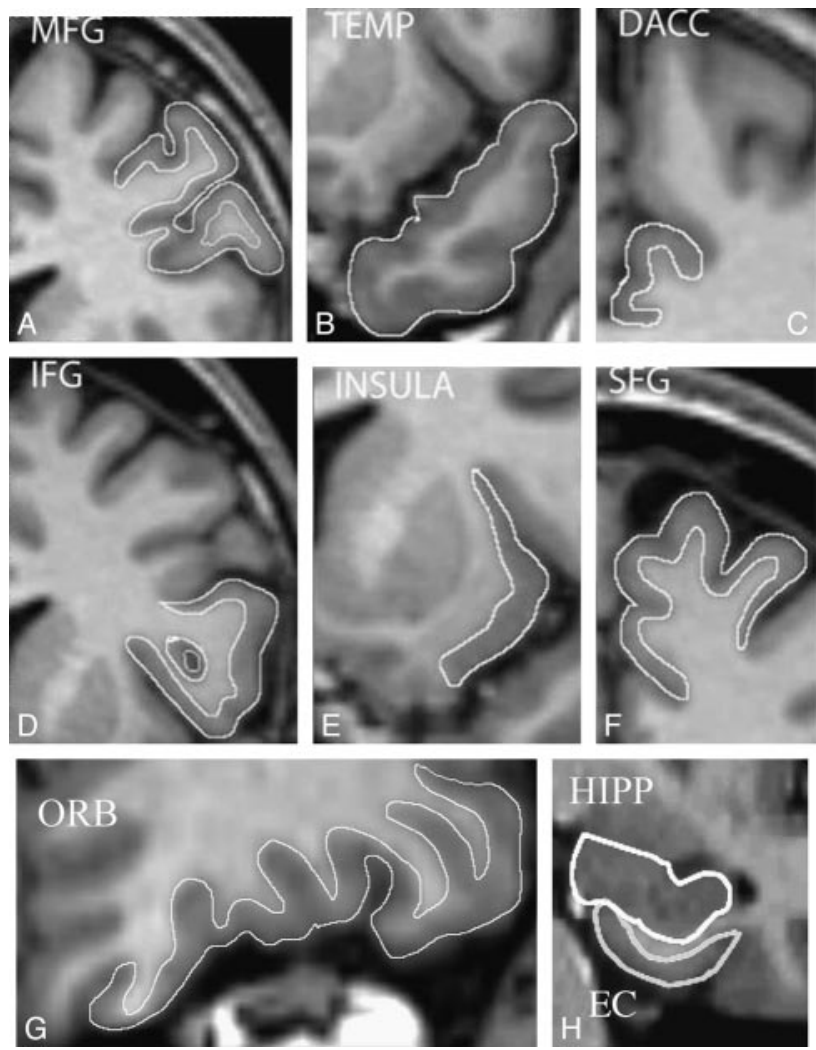

Fig 1. Cortical regions manually outlined on contiguous coronal sections. Note that only gray matter is included in all regions except the TEMP and the HIPP, where both gray and white matter is included.

Sweden) (Fig 1). Regions of interest were traced manually on contiguous coronal sections. The intracranial volume (ICV) was obtained by using a stereologic point-counting technique comprising manual tracing of the ICV on every fourth section, following the landmarks proposed by Eritaia et al. ${ }^{8}$ The volumes of all structures were normalized by the ICV (volume of structure [VLS] / ICV).

\section{Regions of Interest}

Gray matter loss was analyzed in all regions except the temporal pole (TEMP) and hippocampus (HIPP). In the TEMP and HIPP, we focused on the reduction of size in the whole region-that is, the total volume (gray and white matter). We analyzed the following structures:

1) Superior frontal gyrus (SFG) (Brodmann area $[\mathrm{BA}] 6,8,9,9,32)$.

2) Middle frontal gyrus (MFG) (BA 46).

3) Inferior frontal gyrus (IFG) (BA 44, 45).

4) Orbitofrontal cortex (ORB) (BA 10, 11, 12, 47).

5) Insula (BA 13, 14).

6) Rostral anterior cingulate gyrus (RACC) (BA 24).

7) Dorsal anterior cingulate gyrus (DACC) (BA 24).

8) Temporal pole (TEMP) (BA 38).

9) Entorhinal cortex (EC) (BA 28, 34).

10) Hippocampus (HIPP).

Established protocols were used for parcellation of the cortical regions as follows: prefrontal cortex and insula, ${ }^{9} \mathrm{TEMP},{ }^{10} \mathrm{EC},{ }^{11}$ HIPP, ${ }^{12}$ and anterior cingulate gyrus. ${ }^{13}$ For the posterior border of the DACC, however, we followed Fornito et $\mathrm{al}^{14}$ and stopped tracing 1 section after the disappearance of the anterior commissure, moving from anterior to posterior on coronal sections. 
Volumetric data were obtained by 2 raters. Rater 1 (B.B.Z) analyzed the HIPP and the EC. Rater 2 (O.L.) analyzed the cortical and mesocortical regions and the TEMP. Both raters were blind to clinical data.

Reliability of the volumetric data was calculated by the intrarater intraclass correlation coefficient (ICC). The ICC on 15 repeated measurements was 0.91 for the HIPP and 0.94 for the EC. ICC for prefrontal structures on 10 repeated measurements (at least 1 month apart) ranged from 0.91 for the RACC to 0.97 for the SFG. An interrater correlation coefficient was not calculated; however, the delineation of each region on approximately 10 brains was scrutinized and approved by an experienced neuroanatomist (N.B.).

\section{Statistical Analysis}

Discriminant Analysis. The pattern of atrophy was investigated by partial least squares discriminant analysis (PLS-DA), which is a supervised multivariate data analysis method that is part of the SIMCA software (Umetrics AD, Umeå, Sweden). The results from the PLS-DA analysis were visualized by plotting 2 principal components of the model against each other in a scatterplot. This plot illustrates the degree of separation accomplished by the components. Each point in the scatterplot represents 1 patient. Each principal component receives a $\mathrm{Q}^{2}$ value, which describes the statistical significance and the predictive power of that component. $\mathrm{A} \mathrm{Q}^{2}$ value $>0.05$ is considered statistically significant. Sensitivity and specificity were calculated from the predictions made by leave-one-out cross-validation. This means that a number of parallel models are built. Each model leaves 1 individual out. The data from this individual are then introduced into the model; this step results in a predictive value. The theoretically correct predictive value of membership in group 1 is 1 and group 2 is 0 . The cutoff value for the predictions is 0.5 . This means that all individuals with a predictive value $>0.5$ are classified into group 1 , whereas individuals with a value $<0.5$ are classified into group 2 .

Sensitivity and specificity were then calculated as follows: Sensitivity is the number of true-positive predictions divided by the sum of the number of true-positive predictions and the number of falsenegative predictions. Specificity is the number of true-negative predictions divided by the sum of the number of true-negative predictions and the number of false-positive predictions.

The PLS-DA allows us to plot the structures according to their importance for separating the groups. Structures receive a variable of importance (VIP) value. Values $>1$ indicate that the structure is involved above average in the separation of groups.

The $T$ Test with Bonferroni Correction for Multiple Comparisons. Significant differences between individual variables were also identified by a $t$ test. Results were corrected for multiple comparisons by a Bonferroni correction (on Statistica, Version 7.1; Statsoft, Tulsa, Okla). A $P$ value $<.05$ was considered significant.

Every separate measurement of an individual structure was first normalized for ICV by the following formula:

\section{VLS/ICV.}

In comparisons between the subtypes and the controls, this ratio is plotted as a $z$-score. In the comparison between different subtypes, all measured regions are plotted as a ratio of control mean value of VLS/ ICV. The control mean was, therefore, always set to $0 \%$.

The relationship between the localization and rate of atrophy is illustrated by connecting the volumes of anatomically adjacent regions by a horizontal line.

The RACC displayed more anatomic variability than any other investigated region. Because this region was not a differentiating factor in any comparison between subtypes or between a single subtype and controls, it was excluded in the plotting of structures.

Laterality. Hemispheric laterality was investigated by dividing the left-sided structures by the same structures on the right side. We made 5 different calculations of laterality:

1) Frontal laterality: Left (SFG + MFG + IFG + ORB) / Right (SFG + $\mathrm{MFG}+\mathrm{IFG}+\mathrm{ORB})$.

2) Temporal laterality: Left (TEMP + EC + HIPP) / Right (TEMP + $\mathrm{EC}+\mathrm{HIPP})$.

3) Laterality of the insular lobe: Left (insula) / Right (insula).

4) Total laterality: Left (all regions) / Right (all regions).

5) Laterality of a single region: Left (single region) / Right (single region).

Statistical analysis of laterality was made by using the KruskalWallis test followed by Mann-Whitney $U$ tests with a Bonferroni correction. Because patients with SD may display greater asymmetry either on the left or the right, this group was divided into 2 groups for the laterality calculation: SD with more atrophy on the left and SD with more atrophy on the right.

\section{Results}

The characteristics of the pattern of atrophy were investigated by comparing each subtype with the control group.

\section{Pair-Wise Comparisons between Each FTLD Subtype and the Control Group}

SD versus Controls. The $t$ tests with Bonferroni correction revealed that all temporal areas were significantly atrophic in patients with SD (Fig 2A). Left and right TEMPs displayed $>50 \%$ volume loss compared with those in the control mean. There was significant bilateral atrophy in the insula. There was also a tendency to atrophy in the right ORB. Most patients with SD displayed a hemispheric asymmetry of atrophy on inspection. However, this could not be statistically confirmed because patients with predominantly left-sided atrophy cancelled out the effect of laterality in patients with predominantly right-sided atrophy (Fig 3).

FTD versus Controls. Patients with FTD were compared for atrophy with the control group. A $t$ test with a Bonferroni correction revealed bilateral atrophy in the ORB, SFG, and insula. In the temporal lobe, we found bilateral atrophy in EC, HIPP, and TEMP (Fig 2B). Most structures were affected more on the right side.

PNFA versus Controls. Compared with controls, patients with PNFA had significant atrophy in the following structures in the left hemisphere: EC, HIPP, TEMP, insula, SFG, and ORB. Fewer structures were atrophic on the right: EC and ORB (Fig 2C). The PNFA group thus had more left-hemisphere atrophy. A significant difference between groups was also found in the ratio of volume between the left and right insula (left insula/right insula) (Fig 3). The left insula was more atrophic than the right insula in PNFA.

\section{All FTLD Subjects Pooled Together as One Group}

Two regions displayed more anatomic variability than all the others: 2 components of the anterior cingulate gyrus (RACC and DACC) and the IFG. Because of large within-group variability, these regions could not be compared with those in 


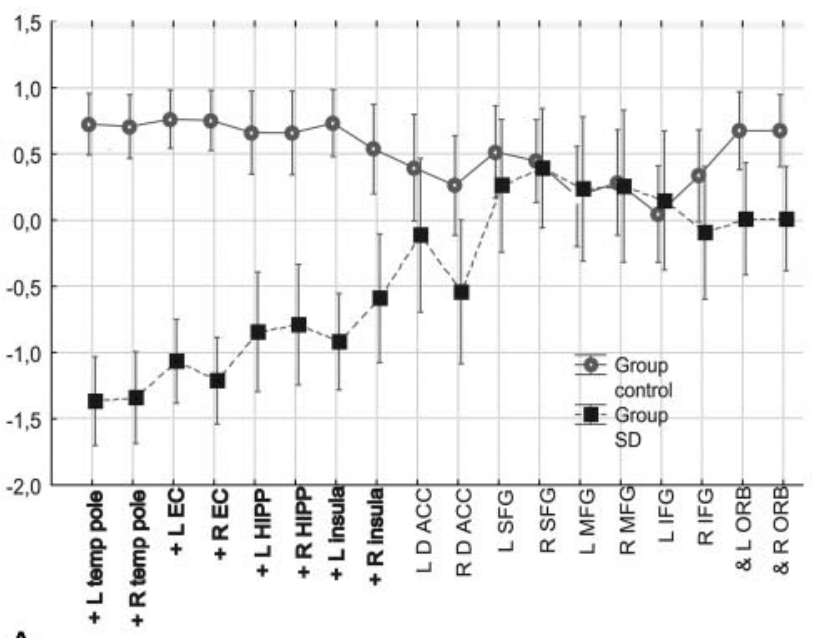

A

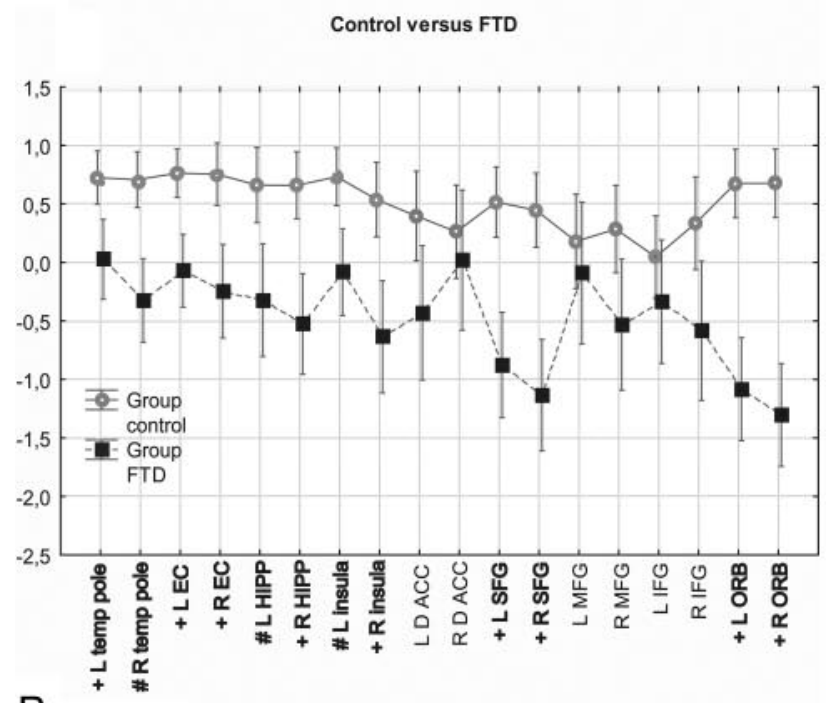

$\mathrm{B}$

R1

Control versus PNFA

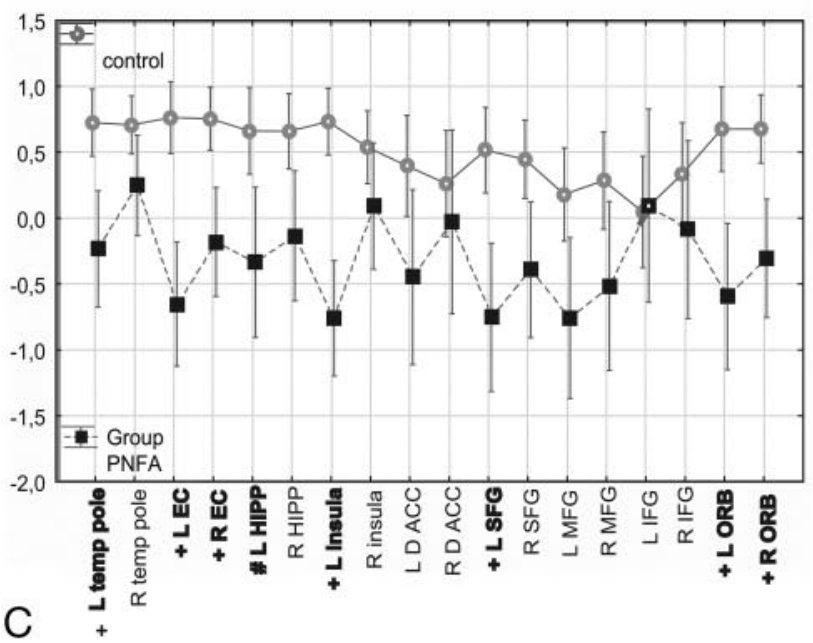

Fig 2. Comparison between FTLD subtypes and controls. $X$-axis denotes volume of interest (VOI). Y-axis denotes VOI divided by intracranial volume normalized as z-scores. To illustrate the overall pattern of cortical atrophy, we connected the structures by a horizontal line. Vertical bars denote the $95 \%$ confidence interval. Plus sign indicates $P<.01$; number sign, $P<.05$; ampersand, $P<.07$ (close to significant); temp pole, temporal pole; L, left: $\mathrm{R}$, Right

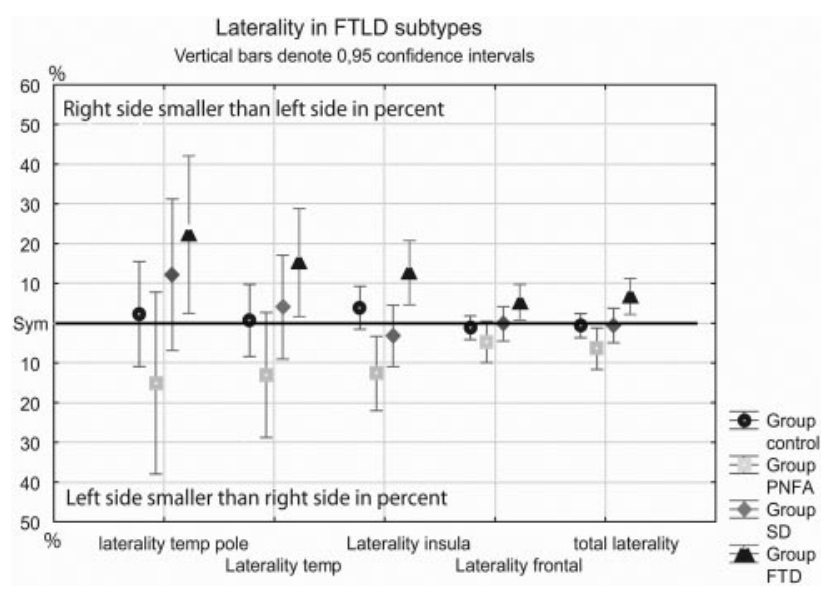

Fig 3. Difference between left- and right-sided volume of interest (VOI) calculated in percentages. FTD versus PNFA: total laterality, $P=.015$. Insula $P<.01$. PNFA versus control insula, $P=.025$. Total indicates the total volume of all measured regions; temp, the volume of all measured temporal regions; temp pole, the volume of temporal pole; Insula, the volume of the insula; frontal, the volume of all frontal regions.

controls in single subtypes. The overall comparison between all FTLD subtypes combined and controls revealed that the RACC was bilaterally atrophic, whereas the DACC was atrophic only on the left (Table 2). No differences between FTLD groups and controls were found in the IFG.

\section{Pairwise Comparisons between the FTLD Subtypes}

FTD versus PNFA: Discriminant Analysis. The results of the discriminant analysis comparing FTD and PNFA are shown in the PLS-DA scatterplot (Fig $4 A$ ). Eleven patients with FTD (11/12) and 8 patients with PNFA (8/9) were correctly classified (sensitivity $92 \%$ and specificity $89 \%$ ) (Table 3 ). Inspection of the VIP plot (Fig 5A) revealed that hemispheric laterality was a crucial factor in the discriminant analysis, because the 4 most important regions in the VIP plot were all on the right. These were all more affected in the FTD group. The next 4 most discriminant regions were all left-sided areas, and except for the left ORB, they were more affected in PNFA group.

FTD versus PNFA: Traditional $t$ Test with Bonferroni Correction. A traditional $t$ test with a Bonferroni correction for multiple comparisons revealed only 1 significant difference between groups: The right ORB was more atrophic in the patients with FTD (Fig 6A).

FTD versus PNFA: Laterality. The laterality difference was confirmed in the calculation of the proportion of the total volume of the left and right side. This revealed a significant difference in which patients with PNFA were more atrophic on the left, whereas patients with FTD were more atrophic on the right (Fig 3). There was also a significant difference in the proportion between the right and left insula (left insula/right insula) (Fig 3).

Table 2: FTLD subjects treated as one group compared with control subjects*

\begin{tabular}{llc}
\hline FTLD & Left Side & Right Side \\
\hline RACC & $P=.047$ & $P<.01$ \\
DACC & $P=.0115$ &
\end{tabular}

Note:-FTLD indiates frontotemporal lobar degeneration; RACC, rostral anterior cingulate gyrus; DACC, dorsal anterior cingulate gyrus.

* Compared by $t$ test with Bonferroni correction for multiple comparisons. 


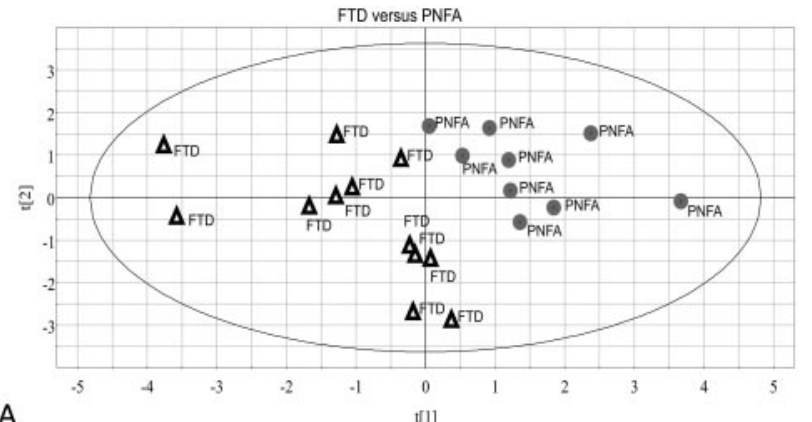

A
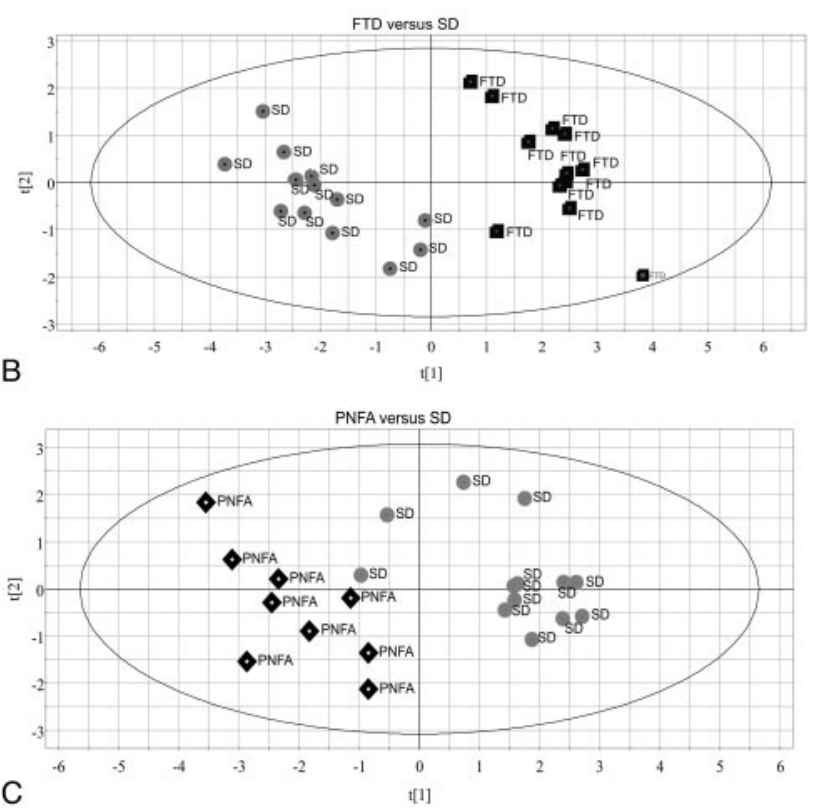

Fig 4. Scatterplots of the PLS-DA models of the comparisons of FTLD subtypes. This plot illustrates the degree of separation between groups. The horizontal scale refers to the first component $(t[1])$, whereas the vertical scale refers to second component $(\mathrm{t}[2])$. $A$, Comparison between FTD and PNFA. B, Comparison between FTD and SD. $C$, Comparison between PNFA and SD.

\begin{tabular}{|c|c|c|c|c|}
\hline Group 1 & Group 2 & $Q^{2 *}$ & Sensitivity & Specificity \\
\hline$\overline{\mathrm{FTD}}$ & PNFA & 0.251 & $92 \%(11 / 12)$ & $89 \%(8 / 9)$ \\
\hline FTD & SD & 0.788 & $100 \%(12 / 12)$ & $100 \%(13 / 13$ \\
\hline SD & PNFA & 0.605 & $86 \%(11 / 13)$ & $100 \%(9 / 9)$ \\
\hline
\end{tabular}

Note:-PLS-DA indicates partial least squares discriminant analysis.

${ }^{*} \mathrm{Q}^{2}$ value $>0.05$ is regarded as significant value for a principal component.

FTD versus SD: Discriminant Analysis. Discriminant analysis comparing FTD and SD is shown in the PLS-DA scatterplot (Fig 4B). All patients were correctly classified in this comparison (sensitivity 100\% [12/12], specificity 100\% [13/ 13]) (Table 3). The VIP plot (Fig 5B) and the plotting of structures (Fig 6B) show that the most important discriminant factor was temporal-versus-frontal atrophy. Eight regions were particularly important for discriminating groups. The 4 temporal regions were all more affected in SD, whereas the 4 frontal regions were all more affected in FTD.

FTD versus SD: Traditional $\boldsymbol{t}$ Test with Bonferroni Correction. A traditional $t$ test with Bonferroni correction revealed that patients with SD displayed more bilateral atrophy in the EC and TEMP and left insula, whereas patients with
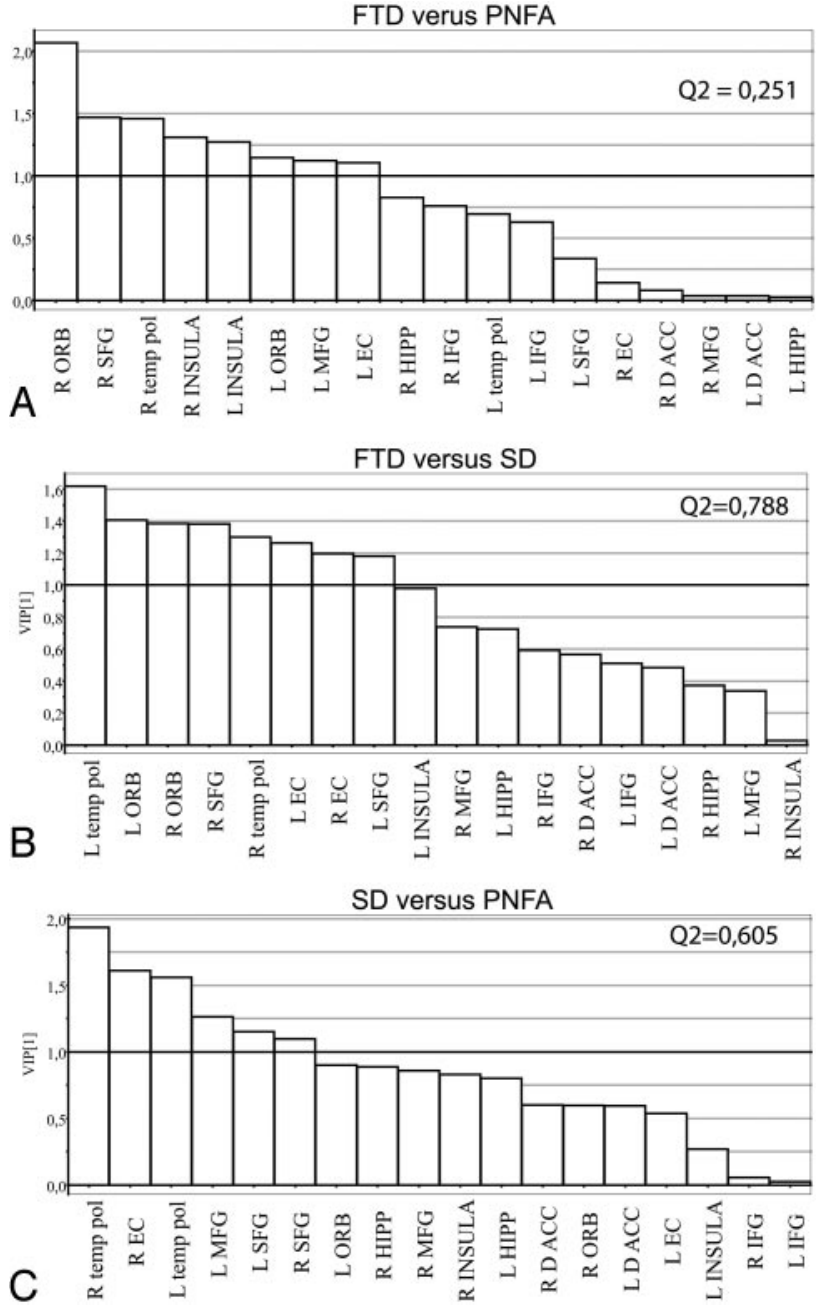

Fig 5. The VIP plots denote the importance of each measured region for separation between the compared subtypes. A variable with a VIP value $>1$ (above the horizontal line) is of above average importance for the separation of the investigated groups. The $x$-axis denotes the regions included in the discriminant analysis. The $y$-axis denotes the VIP value. Temp pol indicates temporal pole; $L$, left; $R$, right.

FTD were more atrophic in the bilateral SFG and ORB (Fig $6 B)$.

FTD versus SD: Laterality. No differences in laterality were found between FTD and SD groups.

SD versus PNFA: Discriminant Analysis. Finally, the discriminant analysis comparing SD and PNFA is shown in the PLS-DA scatterplot (Fig 4C). Two patients with SD were classified as having PNFA in this comparison (sensitivity $86 \%$ [11/ 13], specificity 100\% [9/9]) (Table 3). The VIP plot (Fig 5C) and the plotting of structures (Fig $6 C$ ) revealed that frontalversus-temporal atrophy again was the most important discriminant factor. Three temporal and 3 frontal regions proved particularly important in the VIP plot. The 3 temporal regions were more affected in SD, whereas the 3 frontal regions were more affected in PNFA.

SD versus PNFA: Traditional $t$ Test with Bonferroni Correction. A $t$ test with a Bonferroni correction revealed the following significant differences: The right EC and left and right TEMP were more affected in SD, whereas the left SFG was more atrophied in PNFA (Fig 6C).

SD versus PNFA: Laterality. No differences in laterality were found between the SD and PNFA groups. 
FTD versus PNFA

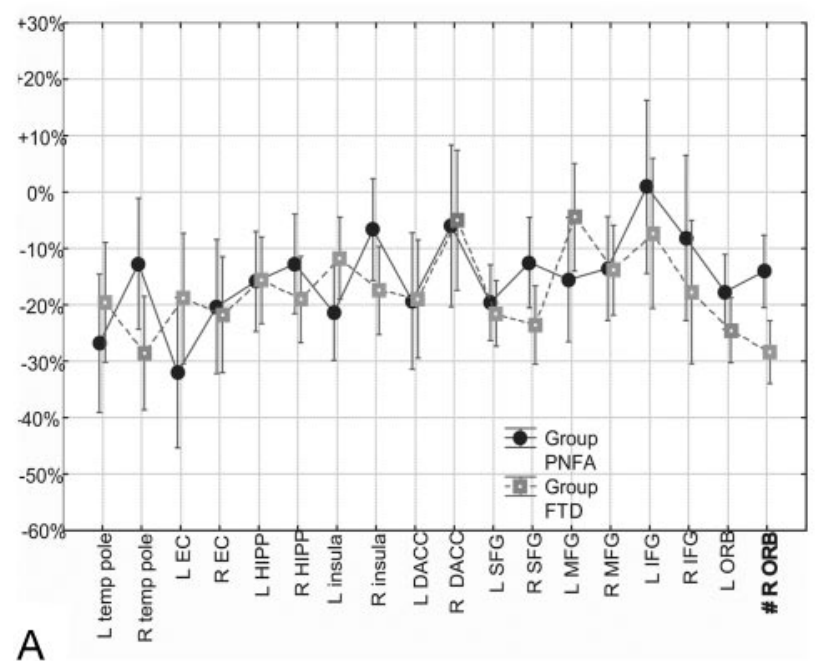

FTD versus SD

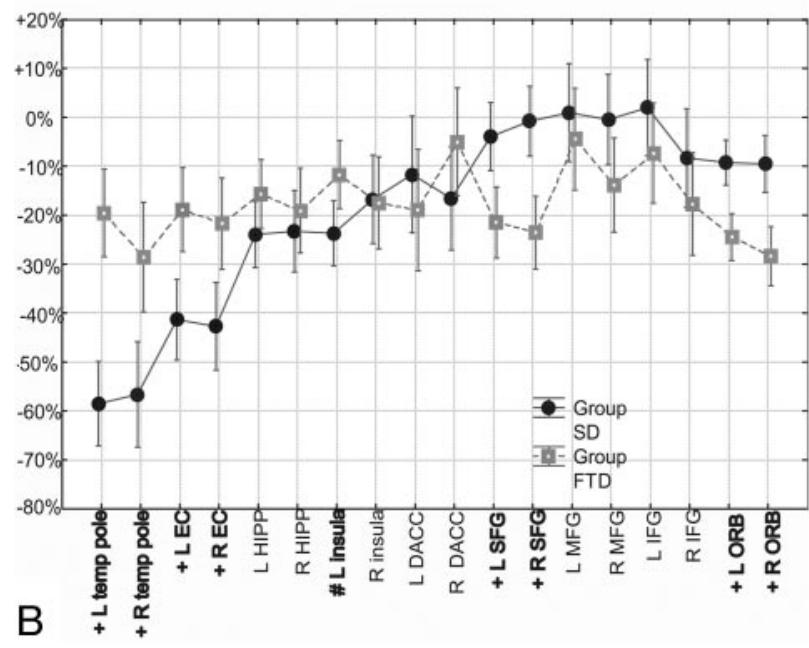

PNFA versus SD

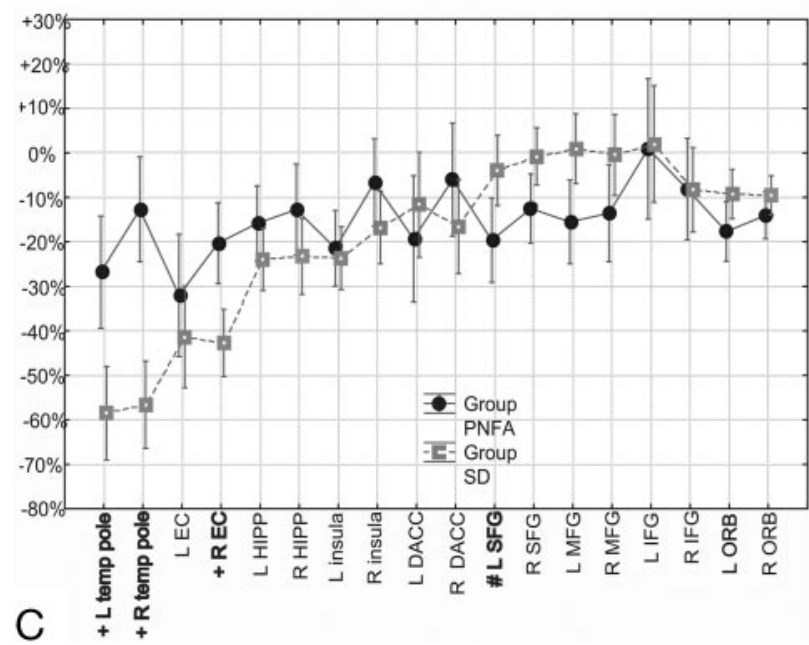

Fig 6. Comparison between FTLD subtypes. X-axis denotes the volume of interest. Y-axis denotes the volume of interest (VOI)/ICV as a ratio of control mean value of VOI/ICV. Control mean value is set to $0 \%$. Vertical bars denote $95 \%$ confidence interval. To emphasis the pattern of atrophy, we connected the structures by a horizontal line. Plus sign indicates $P<.01$; number sign, $P<.05$; temp pole, temporal pole; L. left; $\mathrm{R}$, right.

\section{Discussion}

We investigated whether the 3 clinically defined FTLD subtypes had corresponding characteristic patterns of cortical atrophy. This was largely confirmed by discriminant analysis, according to which a set of 9 cortical regions classified at least $90.5 \%$ of the patients correctly in any pair-wise comparison between 2 FTLD subtypes.

Our results differ from those of Kipps et al, ${ }^{5}$ which were based on a visual rating of MR images from a large case series. Their series might have included cases of "benign behavioral variant FTD" (see below). It is possible that the variability in each subtype was better controlled in the larger sample. However, ordinal data from a visual rating are less sensitive than continuous data derived from careful manual measurement, and expert manual measurement of brain regions remains the gold standard in volumetric studies. Expert manual measurement may thus disclose volumetric pathology in the large proportion of patients with FTD judged healthy on an ordinal rating scale. Alternatively, the cases in Kipps et $\mathrm{al}^{5}$ may belong to a subgroup characterized by normal MR imaging findings and slow progression ("benign behavioral variant FTD"), which, though prevalent in the Cambridge series, was not evident in our sample. ${ }^{15}$

Although our discriminant analysis relied on the combination of all measured cortical regions, some specific regions were found to be particularly important for the discrimination of each subtype. These regions could generally be linked to the specific clinical features displayed by each subtype. The ORB was thus the most vital structure in discriminating FTD from other subtypes. This region has been implicated in behavioral disturbances observed in FTD. ${ }^{16}$ In contrast, in SD, the most effective discriminator was the TEMP. Whereas the left TEMP has been associated with conceptual knowledge, ${ }^{17}$ the right TEMP has been associated with semantic working memory. ${ }^{18}$ The relation between clinical features and brain regions was less distinct in PNFA than in the other subtypes. The left insula was atrophic, and this mesocortical region is involved in language production..$^{19}$ However, because this area was also affected in other subtypes, its discriminative power is limited.

PNFA may be more closely correlated to atrophy of the pars opercularis of the IFG. ${ }^{20}$ In future investigations, it may, therefore, be more rewarding to divide the IFG into pars opercularis, pars triangularis, and pars orbitalis and to measure these parts separately.

Regarding lobar and hemispheric atrophy, we found 2 dichotomies particularly significant: frontal-versus-temporal atrophy and left- versus right-predominant atrophy. Combined, they produce the following diagnostic indices:

SD: atrophy in temporal but little (or no) atrophy in frontal structures in comparison with FTD and PNFA;

PNFA: bilateral atrophy, with left-predominant frontal and temporal atrophy in comparison with FTD.

FTD: bilateral atrophy, with right-predominant frontal and temporal atrophy in comparison with PNFA.

Differing lateralization in FTD (right) and PNFA (left) is consistent with the different clinical features of these disorders. Similarly, Miller et $\mathrm{al}^{21}$ observed that right frontal atrophy is associated with the FTD features of disinhibition and antisocial behavior, whereas the speech-language dis- 
orders of PNFA are associated with lateral areas of the left hemisphere.

Our discriminant models were based on a relatively small number of subjects. In particular, the small number of patients with PNFA ( $n=9)$ might have led to unreliable estimates of sensitivity and specificity, considering the within-group variability of the atrophic pattern in PNFA.

Finally, some methodologic concerns may be addressed. First, a limitation with manual outlining of cortical regions is that the condition of a cortical region may be confused with the variability of that region. The volume of a cortical region may thus be small simply because it is anatomically small, but it may also be small because it is atrophied. The number of subjects included in this investigation should be large enough to rule out the problem of anatomic variability. Two regions, however, displayed so much variability that no difference was found between single subtypes (or between a subtype and controls): the IFG and the anterior cingulate gyrus. In controls and in patients with FTD and SD, the left IFG was approximately $65 \%$ of the size of the MFG. In PNFA, the ratio of IFG/MFG was approximately 75\%. A likely explanation for this is that the IFG on average is distributed over a larger area of the brain in the PNFA group than in the other groups, possibly at the expense of the MFG. Previous anatomic investigations of the IFG have also shown a considerable variability in size and shape of this area. ${ }^{22}$ Nevertheless, despite the confounding factor of anatomic variability, this method has generally proved to be a reliable way of classifying subjects with FTLD into subtypes.

The clinical significance of this study relates to the potential use of the examination of patterns of cortical atrophy for clinical diagnosis, correlation of clinical subtypes with characteristic cortical atrophy relevant to the clinical features of each subtype, and, indirectly, confirmation of the utility of the existing clinical subtypes of FTLD. Further studies are planned to investigate the shape and complexity of the cortical regions, correlations with subcortical structures, and correlations with neuropsychological and behavioral measures.

\section{Conclusions}

We have found that cortical patterns of atrophy are characteristic within subtypes of FTLD. SD shows predominantly temporal atrophy. Although both FTD and PNFA show frontotemporal atrophy, there appear to be lateralized differences with right-sided atrophy in FTD compared with left-sided atrophy in PNFA. Such regional atrophy corresponds to the expected structural basis of the clinical features. Consideration of patterns of cortical morphometric atrophy may inform the diagnosis of FTLD subtypes for future studies and, ultimately, make it possible to contribute significantly to the clinical diagnosis on the basis of MR imaging.

\section{References}

1. Onari K, Spatz H. Anatomische Beiträge zur Lehre von der Pickschen umschriebenen Großhirnrinden-Atrophie ("Picksche Krankheit"). Ges Neurol Psychiatr 1926;101:470-511

2. Neary D, Snowden JS, Gustafson L, et al. Frontotemporal lobar degeneration: a consensus on clinical diagnostic criteria. Neurology 1998;51:1546-54

3. Kertesz A. Frontotemporal dementia: one disease, or many? Probably one, possibly two. Alzheimer Dis Assoc Disord 2005;19(suppl 1):S19-24

4. Grossman M, McMillan C, Moore P, et al. What's in a name: voxel-based morphometric analyses of MRI and naming difficulty in Alzheimer's disease, frontotemporal dementia and corticobasal degeneration. Brain 2004;127(pt 3):628-49. Epub 2004 Feb 4

5. Kipps CM, Davies RR, Mitchell J, et al. Clinical significance of lobar atrophy in frontotemporal dementia: application of an MRI visual rating scale. Dement Geriatr Cogn Disord 2007;23:334-42

6. McMillan C, Gee J, Moore P, et al. Confrontation naming and morphometric analyses of structural MRI in frontotemporal dementia. Dement Geriatr Cogn Disord 2004; 17:320-23

7. Kipps CM, Nestor PJ, Fryer TD, et al. Behavioural variant frontotemporal dementia: not all it seems? Neurocase 2007;13:237-47

8. Eritaia J, Wood SJ, Stewart GW, et al. An optimized method for estimating intracranial volume from magnetic resonance images. Magn Reson Med 2000;44:973-77

9. Crespo-Facorro B, Kim J, Andreasen NC, et al. Cerebral cortex: a topographic segmentation method using magnetic resonance imaging. Psychiatry Res 2000;100:97-126

10. Galton CJ, Patterson K, Graham K, et al. Differing patterns of temporal atrophy in Alzheimer's disease and semantic dementia. Neurology 2001;57:216-25

11. Goncharova II, Dickerson BC, Stoub TR, et al. MRI of human entorhinal cortex: a reliable protocol for volumetric measurement. Neurobiol Aging 2001;22:737-45

12. Jack CR Jr. MRI-based hippocampal volume measurements in epilepsy. Epilepsia 1994;35(suppl 6):S21-29

13. McCormick LM, Ziebell S, Nopoulos P, et al. Anterior cingulate cortex: an MRI-based parcellation method. Neuroimage 2006;32:1167-75

14. Fornito A, Whittle S, Wood SJ, et al. The influence of sulcal variability on morphometry of the human anterior cingulate and paracingulate cortex. Neuroimage 2006;33:843-54

15. Davies RR, Kipps CM, Mitchell J, et al. Progression in frontotempora dementia: identifying a benign behavioral variant by magnetic resonance imaging. Arch Neurol 2006;63:1627-31

16. Peters F, Perani D, Herholz K, et al. Orbitofrontal dysfunction related to both apathy and disinhibition in frontotemporal dementia. Dement Geriatr Cogn Disord 2006;21:373-79

17. Tyler LK, Moss HE. Towards a distributed account of conceptual knowledge. Trends Cogn Sci 2001;5:244-52

18. Martin A, Chao LL. Semantic memory and the brain: structure and processes. Curr Opin Neurobiol 2001;11:194-201

19. Sassa Y, Sugiura M, Jeong H, et al. Cortical mechanism of communicative speech production. Neuroimage 2007;37:985-92

20. Schroeter ML, Raczka K, Neumann J, et al. Towards a nosology for frontotemporal lobar degenerations: a meta-analysis involving 267 subjects. Neuroimage 2007;36:497-510

21. Miller BL, Chang L, Mena I, et al. Progressive right frontotemporal degeneration: clinical, neuropsychological and SPECT characteristics. Dementia 1993;4:204-13

22. Blanton RE, Levitt JG, Peterson JR, et al. Gender differences in the left inferior frontal gyrus in normal children. Neuroimage 2004;22:626-36 